\title{
A DIRICHLET NORM INEQUALITY AND SOME INEQUALITIES FOR REPRODUCING KERNEL SPACES
}

\author{
JACOB BURBEA
}

Abstract. Let $f$ be analytic and of finite Dirichlet norm in the unit disk $\Delta$ with $f(0)=0$. Then, for any $q>0$,

$$
\|\exp f\|_{q}^{2}<\exp \left\{\frac{1}{\pi q} \int_{\Delta}\left|f^{\prime}(z)\right|^{2} d \sigma(z)\right\} \quad(d \sigma(z) \equiv(i / 2) d z \wedge d \bar{z}) .
$$

Equality holds if and only if $f(z)=-q \log (1-z \bar{\zeta})$ for some $\zeta \in \Delta$. Here, for $g(z)=\sum_{n=0}^{\infty} b_{n} z^{n}$, analytic in $\Delta$,

$$
\|g\|_{q}^{2} \equiv \sum_{n=0}^{\infty} \frac{n !}{(q)_{n}}\left|b_{n}\right|^{2}
$$

where $(q)_{0}=1$ and $(q)_{n}=q(q+1) \cdots(q+n-1)$ for $n>1$. This also extends with a substantially easier proof, a result of Saitoh concerning the case of $q>1$. In addition, a sharp norm inequality, valid for two functional Hilbert spaces whose reproducing kernels are related via an entire function with positive coefficients, is established.

1. Introduction. Let $\Delta$ be the unit disk in the complex plane and let $q$ be a fixed positive real number. We shall write $d \sigma(z)=2^{-1} i d z \wedge d \bar{z}$ for the area Lebesgue measure. Quite recently, Saitoh [5] has established the following result.

THEOREM A. Assume $q \geqslant 1$ and let $f$ be an analytic function in $\Delta$ with a finite Dirichlet norm and $f(0)=0$. Then

$$
\frac{q-1}{\pi} \int_{\Delta}|\exp f(z)|^{2}\left(1-|z|^{2}\right)^{q-2} d \sigma(z) \leqslant \exp \left\{\frac{1}{\pi q} \int_{\Delta}\left|f^{\prime}(z)\right|^{2} d \sigma(z)\right\}
$$

$$
q>1
$$

and

$$
\frac{1}{2 \pi} \int_{\partial \Delta}|\exp f(z)|^{2}|d z| \leqslant \exp \left\{\frac{1}{\pi} \int_{\Delta}\left|f^{\prime}(z)\right|^{2} d \sigma(z)\right\} \quad(q=1) .
$$

Equality holds if and only if $f(z)$ is of the form $-q \log (1-z \bar{\zeta})$ for some $\zeta \in \Delta$.

Saitoh [5] obtained this result by appealing to the general theory of reproducing kernels of Aronszajn [1]. His proof concerning the equality statement, however, is difficult and rather lengthy. In this paper we shall give a shorter proof of Theorem $A$ and in so doing even extend the theorem to the case of $0<q<1$. In the extreme case of $0<q<1$, which is not covered by Saitoh [5], the integrals on the

Received by the editors July 25, 1980 and, in revised form, November 4, 1980.

1980 Mathematics Subject Classification. Primary 30C40, 46E20.

Key words and phrases. Dirichlet norm, reproducing kernel spaces.

() 1981 American Mathematical Society 0002-9939/81/0000-0462/\$02.75 
left-hand sides of (1.1) or (1.2) have to be replaced by a suitable Hardy-space norm. We shall also establish a sharp inequality which is valid for general reproducing kernel spaces.

2. A Dirichlet norm inequality. We denote by $H(\Delta)$ the class of all analytic functions in the unit disk $\Delta$. For $q>0$, we write

$$
D_{q}=\left\{f \in H(\Delta): f(0)=0,\|f\|_{D_{q}}<\infty\right\}
$$

with

$$
\|f\|_{D_{q}}^{2}=\frac{1}{\pi q} \int_{\Delta}\left|f^{\prime}(z)\right|^{2} d \sigma(z)=\frac{1}{q} \sum_{n=1}^{\infty} n\left|a_{n}\right|^{2}
$$

and where

$$
f(z)=\sum_{n=1}^{\infty} a_{n} z^{n}, \quad z \in \Delta .
$$

We shall call $D_{q}$ the $q$-Dirichlet-space, and we observe that it is a functional Hilbert space of analytic functions in $\Delta$ with the reproducing kernel

$$
k_{q}(z, \bar{\zeta})=-q \log (1-z \bar{\zeta}), \quad z, \zeta \in \Delta .
$$

Another related functional Hilbert space is the so-called (generalized) $q$-Hardyspace $H_{q}$. This is the space of analytic functions $g$ in $\Delta$ whose norm is given by

$$
\|g\|_{q}^{2}=\sum_{n=0}^{\infty} \frac{n !}{(q)_{n}}\left|b_{n}\right|^{2} ; \quad g(z)=\sum_{n=0}^{\infty} b_{n} z^{n}, \quad z \in \Delta .
$$

Here $(q)_{0}=1$ and $(q)_{n}=q(q+1) \cdots(q+n-1)$ for $n>1$. The reproducing kernel of $H_{q}$ is $K_{q}(z, \bar{\zeta})=(1-z \bar{\zeta})^{-q}$, and we note the relationship $K_{q}(z, \bar{\zeta})=$ $\exp \left[k_{q}(z, \bar{\zeta})\right], z, \zeta \in \Delta$.

The space $H_{q}, q>0$, can be also defined for any simply-connected domain $(\neq C)$ via an isometry induced by conformal mappings (see [3] for more details). Furthermore, the norm in (2.2) can be identified as an $L_{2}$-norm of a certain Laplace transform (see [3]). For $q>1$ the norm can be also realized in a form which appears frequently in function theory. In fact

$$
\begin{aligned}
\|g\|_{q}^{2} & =\frac{q-1}{\pi} \int_{\Delta}|g(z)|^{2}\left(1-|z|^{2}\right)^{q-2} d \sigma(z), \quad q>1, \\
\|g\|_{1}^{2} & =\frac{1}{2 \pi} \int_{\partial \Delta}|g(z)|^{2}|d z|
\end{aligned}
$$

where in the last integral, $g$ stands for the nontangential boundary values of the analytic function $g(z)$ in $\Delta$. Thus, $H_{1}$ is the usual Hardy-Szegö space, $H_{2}$ is the Bergman space and $H_{q}, q>1$, is the Bergman-Selberg space [3]. The norm of $H_{q}$, $0<q<1$, does not admit such a simple realization. However, this fact is not crucial for we shall only use (2.2) as the norm of $g \in H_{q}, q>0$.

The following, therefore, constitutes an extension of Theorem A of Saitoh [5]. 
THEOREM 1. Let $q>0$ and let $f \in D_{q}$. Then

$$
\|\exp f\|_{q}^{2} \leqslant \exp \|f\|_{D_{q}}^{2}
$$

with equality holding if and only if $f$ is of the form $f(z)=k(z, \bar{\zeta})=-q \log (1-z \bar{\zeta})$ for some $\zeta \in \Delta$.

The main difficulty in this theorem is in the necessity of the equality statement. The proof of Saitoh [5], who confined himself only to the case of $q>1$, is rather difficult and involved. We shall give another proof of Theorem 1 which is easier than that of Saitoh [5] and also extends it to $0<q<1$.

Let

$$
f(z)=\sum_{n=0}^{\infty} a_{n} z^{n}
$$

and let

$$
g(z)=\exp [f(z)]=\sum_{n=0}^{\infty} b_{n} z^{n}
$$

be its exponential transform. Then

$$
b_{n}=\frac{1}{n} \sum_{k=0}^{\infty} k a_{k} b_{n-k} ; \quad n=1,2, \ldots\left(b_{0}=1\right) .
$$

3. Proof of Theorem 2. In view of (2.1), (2.2), (2.3) and (2.4), Theorem 1 is equivalent to the following lemma.

LEMMA 1. Let the notation of (2.3) and (2.4) apply and let $q>0$. Then

$$
\sum_{n=0}^{\infty} \frac{n !}{(q)_{n}}\left|b_{n}\right|^{2} \leqslant \exp \left\{\frac{1}{q} \sum_{n=1}^{\infty} n\left|a_{n}\right|^{2}\right\}
$$

if the right-hand side is finite. Equality holds if and only if $a_{n}=q n^{-1} \bar{\zeta}^{n}(n>1)$ and $b_{n}=(n !)^{-1}(q)_{n} \bar{\zeta}^{n}(n \geqslant 0)$ for some $\zeta \in \Delta$.

Proof. For $r \in[0,1)$, define

$$
A(r)=\frac{1}{q} \sum_{n=1}^{\infty} n\left|a_{n}\right|^{2} r^{n}, \quad B(r)=\sum_{n=0}^{\infty} \frac{n !}{(q)_{n}}\left|b_{n}\right|^{2} r^{n} ; \quad r \in[0,1) .
$$

By (2.4) and the Cauchy-Schwarz inequality

$$
\begin{aligned}
\left|b_{n}\right|^{2} & =\frac{1}{n^{2}}\left|\sum_{k=1}^{n} k a_{k}\left[\frac{(n-k) !}{(q)_{n-k}}\right]^{1 / 2} b_{n-k}\left[\frac{(q)_{n-k}}{(n-k) !}\right]^{1 / 2}\right|^{2} \\
& \leqslant \frac{1}{n^{2}}\left(\sum_{k=1}^{n} k^{2}\left|a_{k}\right|^{2} \frac{(n-k) !}{(q)_{n-k}}\left|b_{n-k}\right|^{2}\right) \sum_{k=1}^{n} \frac{(q)_{n-k}}{(n-k) !} \quad(n>1) .
\end{aligned}
$$

On the other hand we have the identity

$$
\sum_{k=1}^{n} \frac{(q)_{n-k}}{(n-k) !}=\sum_{k=0}^{n-1} \frac{(q)_{k}}{k !}=\frac{1}{q} \frac{(q)_{n}}{(n-1) !}
$$


and therefore

$$
n \frac{n !}{(q)_{n}}\left|b_{n}\right|^{2} \leqslant \frac{1}{q} \sum_{k=1}^{n} k^{2}\left|a_{k}\right|^{2} \frac{(n-k) !}{(q)_{n-k}}\left|b_{n-k}\right|^{2} \quad(n>1) .
$$

This shows that $B^{\prime}(r)<A^{\prime}(r) B(r)$ or $[\log B(r)]^{\prime}<A^{\prime}(r)$. Therefore, since $A(0)=0$ and $B(0)=1$,

$$
\log B(r)=\int_{0}^{r}[\log B(x)]^{\prime} d x<\int_{0}^{r} A^{\prime}(x) d x=A(r)
$$

and (3.1) follows by letting $r \rightarrow 1-0$. In view of $[\log B(r)]^{\prime}<A^{\prime}(r), r \in[0,1)$, equality in (3.1) holds if and only if $B^{\prime}(r)=A^{\prime}(r) B(r)$ for each $r \in[0,1)$ which is equivalent to having equality in (3.3) for every $n>1$. This is, clearly, equivalent to an existence of $\lambda_{n} \in \mathbf{C}$ so that

$$
k a_{k} b_{n-k}=\lambda_{n} \frac{(q)_{n-k}}{(n-k) !} ; \quad k=1,2, \ldots, n .
$$

Putting $k=n$ in (3.4) results in $\lambda_{n}=n a_{n}$. Also, using (2.4), (3.4) and the identity (3.2), we have $\lambda_{n}=n ! b_{n} q /(q)_{n}$ and therefore

$$
n a_{n}=q \frac{n !}{(q)_{n}} b_{n} ; \quad n=1,2, \ldots
$$

The only solution of (3.5) subject to (2.4) is

$$
a_{n}=\frac{q}{n} \bar{\zeta}^{n}, \quad \bar{\zeta} \equiv \frac{a_{1}}{q} ; \quad n=1,2, \ldots,
$$

and, therefore, $b_{n}=(q)_{n} \bar{\zeta}^{n} / n$ ! for $n \geqslant 0$. Indeed, for $n>1$, in view of (2.4) and (3.5),

$$
\begin{aligned}
n a_{n} & =q \frac{n !}{(q)_{n}} \frac{1}{n} \sum_{k=0}^{n-1}(n-k) a_{n-k} b_{k} \\
& =q \frac{n !}{(q)_{n}} \frac{1}{n}\left[n a_{n}+\sum_{k=1}^{n-1}(n-k) a_{n-k} b_{k}\right] \\
& =q \frac{n !}{(q)_{n}} \frac{1}{n}\left[n a_{n}+\frac{1}{q} \sum_{k=1}^{n-1} \frac{k(n-k)}{k !} a_{k} a_{n-k}(q)_{k}\right]
\end{aligned}
$$

This results in

$$
a_{n}\left[n-n ! \frac{q}{(q)_{n}}\right]=\frac{n !}{n} \frac{1}{(q)_{n}} \sum_{k=1}^{n-1} \frac{k(n-k)}{k !} a_{k} a_{n-k}(q)_{k} .
$$

To prove (3.6), we use induction on $n$. For $n=1,(3.6)$ is a triviality while for $n>1$, by (3.7) and the inductive assumption,

$$
a_{n}\left[n-n ! \frac{q}{(q)_{n}}\right]=\frac{n !}{n} \frac{1}{(q)_{n}} q^{2 \xi^{n}} \sum_{k=1}^{n-1} \frac{(q)_{k}}{k !}, \quad n>1 .
$$

Therefore, by (3.2),

$$
a_{n}\left[n-n ! \frac{q}{(q)_{n}}\right]=\frac{n !}{n} \frac{1}{(q)_{n}} q^{2 \xi^{n}}\left[\frac{1}{q} \frac{(q)_{n}}{(n-1) !}-1\right], \quad n>1,
$$


or

$$
a_{n}\left[n-n ! \frac{q}{(q)_{n}}\right]=\frac{q}{n} \bar{\zeta}^{n}\left[n-n ! \frac{q}{(q)_{n}}\right], \quad n>1 .
$$

However, the common factor in the square bracket is positive for $n>1$ and $q>0$, and (3.6) follows at once. Since the right-hand side of (3.1) is finite, $\zeta$ of (3.6) must satisfy $|\zeta|<1$. This concludes the proof of the lemma.

4. Extensions to reproducing kernel spaces. The inequality and the sufficient condition in the equality statement of Theorem 1 exhibit a phenomenon which is valid in any two functional Hilbert spaces whose reproducing kernels are related via an entire function with positive coefficients. We shall briefly discuss this possibility, keeping in mind Theorem 1 as a motivation.

Let $k=k(z, \bar{\zeta})$ be a positive-definite Hermitian kernel where $z, \zeta$ vary in a fixed base set $D$. Then, as is well known [1], the kernel $k$ determines a uniquely defined Hilbert space $H_{k}$ of functions on $D$ with norm $\|f\|_{k}$ and for which $k$ is its reproducing kernel. Althouth not necessary, we shall always assume that $k$ is truly Hermitian in the sense that it is complex-valued. In this case, $H_{k}$ is a Hilbert space over the complex field $\mathbf{C}$.

Let $F$ be a nonconstant entire function

$$
F(t)=\sum_{n=0}^{\infty} r_{n} t^{n} ; \quad r_{n}>0
$$

and consider the positive-definite Hermitian kernel

$$
K(z, \bar{\zeta})=F[k(z, \bar{\zeta})]=\sum_{n=0}^{\infty} r_{n}[k(z, \bar{\zeta})]^{n} ; \quad z, \zeta \in D
$$

on $D$. This kernel determines another Hilbert space $H_{K}$ of functions $g$ on $D$ with norm $\|g\|_{K}$ and for which $K$ is its reproducing kernel. The relationship between these two Hilbert spaces is

$$
H_{K}=F\left(H_{k}\right)=\bigoplus_{n=0}^{\infty} r_{n}\left[\left(H_{k}\right)_{\otimes}^{n}\right]
$$

where $\left[\left(H_{k}\right)_{\otimes}^{0}\right]_{r}=\left(H_{k}\right)_{\otimes}^{0}=\mathrm{C},\left[\left(H_{k}\right)_{\otimes}^{1}\right]_{r}=\left(H_{k}\right)_{\otimes}^{1}=H_{k}$ and $\left[\left(H_{k}\right)_{\otimes}^{n}\right]_{r}, n>2$, is described below. Let $\left\{\phi_{\alpha}\right\}, \alpha \in \Lambda$, be an orthonormal basis for $H_{k}$, then the space $\left(H_{k}\right)_{\otimes}^{n}, n \geqslant 2$, is the Hilbert space of functions $f$ on $D^{n}$

$$
f\left(z_{1}, z_{2}, \ldots, z_{n}\right)=\sum A_{\alpha_{1} \alpha_{2}} \cdots \alpha_{n} \phi_{\alpha_{1}}\left(z_{1}\right) \phi_{\alpha_{2}}\left(z_{2}\right) \cdots \phi_{\alpha_{n}}\left(z_{n}\right)
$$

with the norm

$$
\|f\|_{k: \otimes n}=\left\{\sum\left|A_{\alpha_{1} \alpha_{2} \ldots \alpha_{n}}\right|^{2}\right\}^{1 / 2}<\infty ; \quad\left(\alpha_{1}, \alpha_{2}, \ldots, \alpha_{n}\right) \in \Lambda^{n} .
$$

The space $\left[\left(H_{k}\right)_{\otimes}^{n}\right]_{r}(n \geqslant 2)$ is formed by restricting functions in $\left(H_{k}\right)_{\otimes}^{n}$ to the diagonal set of $D^{n}$. Here, the norm of $f \in\left[\left(H_{k}\right)_{\otimes}^{n}\right]_{r}$ is given by $\min \|h\|_{k: \otimes n}$ for all $h$, whose restriction to the diagonal of $D^{n}$ is $f$. For additional details, see Aronszajn [1] (see also Saitoh [5]). 
An element $g \in H_{K}$ can therefore be written as

$$
g(z)=\sum_{n=0}^{\infty} r_{n} f_{n}(z) ; \quad z \in D,
$$

where $f_{0}(z)$ is a constant, $f_{1} \in H_{k}$ and $f_{n} \in\left[\left(H_{k}\right)_{\otimes}^{n}\right]_{r}$. The norm is given by

$$
\|g\|_{K}^{2}=\min \left\{\sum_{n=0}^{\infty} r_{n}\left\|f_{n}\right\|_{k: \otimes n, r}^{2}\right\}
$$

where the minimum is taken over all representations (4.1) of $g \in H_{K}$. Let now $f \in H_{k}$. Then

$$
F[f(z)]=\sum_{n=0}^{\infty} r_{n}[f(z)]^{n}, \quad z \in D,
$$

and clearly $[f(z)]^{n} \in\left[\left(H_{k}\right)_{\otimes}^{n}\right]_{r}, n>0$. Consequently, using (4.2), we obtain

$$
\|F \circ f\|_{K}^{2}<\sum_{n=0}^{\infty} r_{n}\|f\|_{k}^{2 n}=F\left(\|f\|_{k}^{2}\right) \text {. }
$$

We therefore have the following theorem.

THEOREM 2. Let $F$ be a nonconstant entire function with nonnegative coefficients. Let $H_{k}$ and $H_{K}$ be Hilbert spaces of functions on the same base set $D$ with reproducing kernels $k$ and $K$, respectively. Assume further that $K(z, \bar{\zeta})=F[k(z, \bar{\zeta})]$ for every $z, \zeta \in D$. Then

$$
\|F \circ f\|_{K}^{2} \leqslant F\left(\|f\|_{k}^{2}\right) ; \quad f \in H_{k} .
$$

Equality in (4.3) occurs when $f$ is of the form $f(z)=k(z, \bar{\zeta})$ for all $z \in D$ and some $\zeta \in D$.

In order to prove the necessity of the equality statement one must add some additional structure to the Hilbert spaces involved. One such a requirement is, for example, that $H_{k}$ is a space of analytic functions in a bounded domain $D$ of $\mathbf{C}^{n}$. One is then able to choose a particularly convenient orthonormal basis. Even with these assumptions the establishment of the necessity of the equality statement is still technically difficult (see [2], [4] for details). We shall not pursue this point in its full generality. We, however, note that Theorem 1 is specialized here in the assumptions that $D$ is the unit disk $\Delta$ and $F$ is the entire function $F(t)=\exp (t)$. The last theorem may also be applied to spaces of square summable series described below.

Let $\Delta_{\rho}=\{z \in \mathbf{C}:|z|<\rho\}, \rho=1, \infty$, and assume that $\phi(z)=\sum_{n=0}^{\infty} c_{n} z^{n}, c_{n}>$ 0 , is analytic in $\Delta_{\rho}$. Let $H\left(\Delta_{\rho}\right)$ denote the class of analytic functions in $\Delta_{\rho}$ and write

$$
H_{\phi}=\left\{f \in H\left(\Delta_{\rho}\right): f(z)=\sum_{n=0}^{\infty} a_{n} z^{n}, \sum_{n=0}^{\infty} c_{n}^{-1}\left|a_{n}\right|^{2}<\infty\right\} .
$$

Clearly, this is a Hilbert space with the norm

$$
\|f\|_{\phi}=\left\{\sum_{n=0}^{\infty} c_{n}^{-1}\left|a_{n}\right|^{2}\right\}^{1 / 2}
$$


We have

$$
f(\zeta)=\sum_{n=0}^{\infty} a_{n} \zeta^{n}=\sum_{n=0}^{\infty} \frac{a_{n} c_{n} \zeta^{n}}{c_{n}}=(f(z), \phi(z \bar{\zeta}))_{\phi}
$$

which means that $K(z, \bar{\zeta})=\phi(z \bar{\zeta})$ is the reproducing kernel of $H_{\phi}$. The sequence $\left\{\sqrt{c_{n}} z^{n}\right\}, n \geqslant 0$, is, of course, an orthonormal basis for $H_{\phi}$. It is also clear that $\phi_{m}(z \bar{\zeta})=\sum_{n=m}^{\infty} c_{n}(z \bar{\zeta})^{n}, m \geqslant 1$, is the reproducing kernel of the closed subspace of $H_{\phi}$

$$
H_{\phi_{m}}=\left\{f \in H_{\phi}: f(0)=f^{\prime}(0)=\cdots=f^{(m-1)}(0)=0\right\} .
$$

Therefore, by making the identification $\phi_{0}=\phi$, we may allow some of the $c_{n}$ in the definition of $H_{\phi}$ to be zero with the obvious interpretation.

With this notation, Theorem 2 admits the following corollary.

COROLlARY 1. Let $F$ be a nonconstant entire function with nonnegative coefficients. Let $\phi$ be as before and write

$$
\psi(z)=F[\phi(z)]=\sum_{n=0}^{\infty} d_{n} z^{n} \quad\left(z \in \Delta_{\rho}, d_{n}>0\right) .
$$

Let $f(z)=\sum_{n=0}^{\infty} a_{n} z^{n} \in H_{\phi}$ and consider its transform

$$
F[f(z)]=\sum_{n=0}^{\infty} b_{n} z^{n}, \quad z \in \Delta_{\rho} .
$$

Then $F \circ f \in H_{\psi}$ and

$$
\sum_{n=0}^{\infty} d_{n}^{-1}\left|b_{n}\right|^{2}<F\left(\sum_{n=0}^{\infty} c_{n}^{-1}\left|a_{n}\right|^{2}\right) .
$$

Equality occurs when $f(z)=\phi(z, \bar{\zeta})$ for some $\zeta \in \Delta_{\rho}$ or when $a_{n}=c_{n} \bar{\zeta}^{n}\left(b_{n}=d_{n} \bar{\zeta}^{n}\right)$.

Many interesting inequalities arise from (4.4) by a suitable choice of the functions $\phi$ and $F$. These inequalities are then interpreted as norm inequalities for Hilbert spaces of entire functions when $\rho=\infty$ or of analytic functions in $\Delta$ when $\rho=1$ (see [3] for details). As an example, we take the unit disk $\Delta$ and $q>0$. We choose $\phi(z)=-q \log (1-z)$ and, therefore, $c_{0}=0$ and $c_{n}=q^{n-1}$ for $n>1$. The Hilbert space $H_{\phi}$ induced by $\phi$ is then the $q$-Dirichlet-space $D_{q}$ whose norm is as in (2.1), and, $k_{q}(z, \bar{\zeta})=\phi(z \bar{\zeta})$. We also take $F(t)=\exp (t)$ and therefore $\psi(z)=$ $(1-z)^{-q}$. The Hilbert space $H_{\psi}$ is now the $q$-Hardy-space $H_{q}$ whose norm is as in (2.2), and $K_{q}(z, \bar{\zeta})=\psi(z \bar{\zeta})$. The assertions of Theorem 1 , short of the necessity of the equality statement, are clearly deductible from Corollary 1.

\section{REFERENCES}

1. N. Aronszajn, Theory of reproducing kernels, Trans. Amer. Math. Soc. 68 (1950), 337-404.

2. J. Burbea, On the Bergman metrics and their indicatrixes, Duke Math. J. 39 (1972), 9-18.

3. __ Total positivity of certain reproducing kernels, Pacific J. Math. 67 (1976), 101-130.

4. The curvatures of the analytic capacity, J. Math. Soc. Japan 29 (1977), 755-761.

5. S. Saitoh, Some inequalities for analytic functions with a finite Dirichlet integral on the unit disc, Math. Ann. 246 (1979), 69-77.

Department of Mathematics, University of Ptttsburgh, Pittsburgh, Pennsylvania 15260 